\title{
Novel Stable Resonator Concept for Efficient Axisymmetric Output from Lasers with a Planar Gain Medium
}

\author{
J. I. Mackenzie and W.A. Clarkson \\ Optoelectronics Research Centre, University of Southampton, Highfield, Southampton SO17 1BJ, U.K. \\ iim@orc.soton.ac.uk
}

\begin{abstract}
A novel resonator concept that produces axisymmetric output from a thin-planar gain medium with a highly elliptical pump cross-section is described. Preliminary studies of this power-scalable approach using a Nd:YLF slab laser are presented.
\end{abstract}

OCIS codes: (140.3410) Laser resonators; (140.3300) Laser beam shaping

\section{Introduction}

Traditional high-power laser designs aim to overcome the heat generated within the laser medium that causes efficiency loss, and potentially limited operational lifetime. The focus of attention has been on novel pumping schemes and gain media geometries that minimise thermal loading and/or its deleterious effects. Three competing laser architectures (thin-slab, thin-disc and fibre) have emerged as the most promising schemes for alleviating the problems associated with these thermal effects. The planar geometry, in addition to excellent thermal management, offers the flexibility to optimise the active ion concentration and host material dimensions, and accordingly the round-trip gain, with the potential for producing high pulse energies as well as high average powers. Despite these benefits, slabs lasers typically suffer poor in-plane beam quality when using standard stable cavities, with previously reported solutions including multi-pass resonator designs [1], or the use of intracavity cylindrical optics [2].

In this paper, we present preliminary findings for a proof-of-principle investigation of a novel, low-loss, stableresonator concept that transforms a highly elliptical cavity mode, providing excellent overlap with the thin-planar excitation volume, into an axially symmetric output beam. Demonstrated using a diode-bar end-pumped Nd:YLF thin-slab gain medium with a pump focal-spot aspect ratio of 10:1, good laser efficiency and beam quality resulted. The simplicity of this resonator concept is a key advantage to our approach. It has the potential to not-only enable brightness scaling of thin slab-lasers with a convenient circular output beam, but could well challenge the current dominance of thin-disc and fibre lasers. Moreover, it offers a unique mode of operation, the capacity to generate high pulse-energies through gain engineering, not available to these other geometries.

\section{Resonator concept}

Our approach employs a simple optical arrangement comprising two high reflectivity mirrors, which are aligned so that the sagittal plane of the first mirror (A) becomes the tangential plane of the second (B), simply by tilting them about orthogonal axes, $\mathrm{x}$ and $\mathrm{y}$ as shown in Fig. 1. Thereby, exploiting the inherent relative astigmatism exhibited by off-axis reflections from a curved surface due to the difference in focal length for the two incident planes, i.e. $\mathrm{f}^{\mathrm{tan}}=\mathrm{R} \cos (\theta) / 2$ and $\mathrm{f}^{\mathrm{sag}}=\mathrm{R} /(2 \cos (\theta))$, where $\theta$ is the incidence angle, and $\mathrm{R}$ the mirror radius. If the angles of

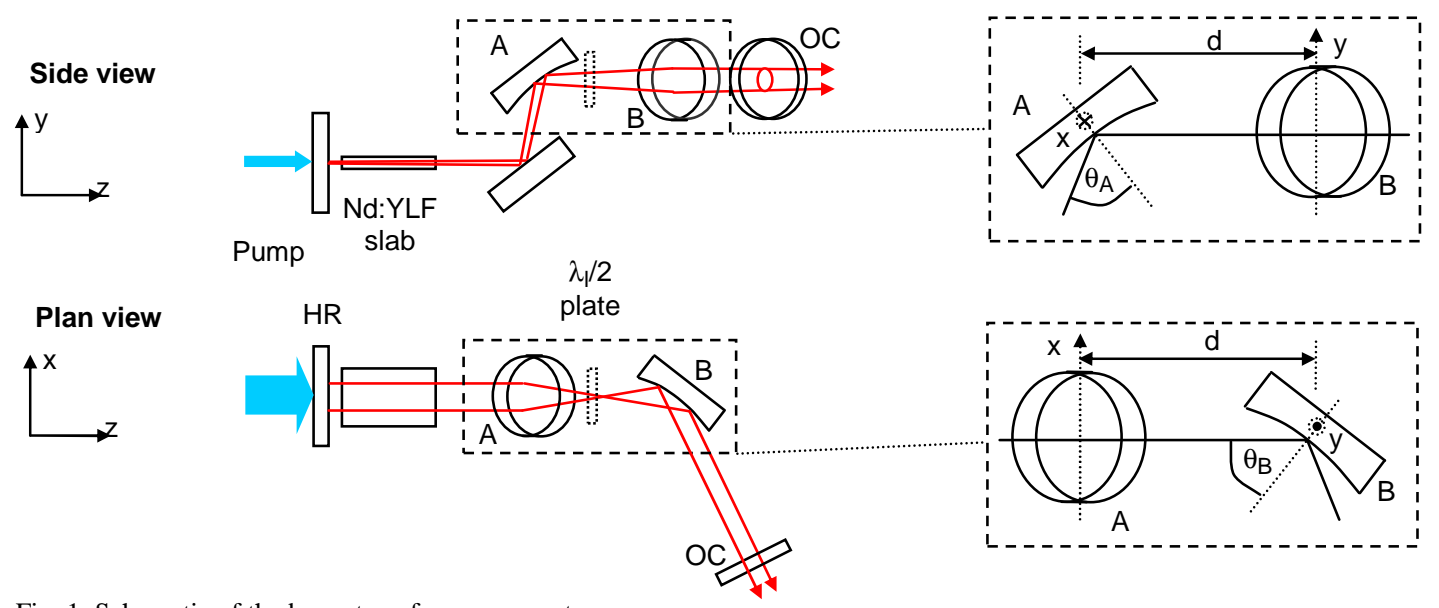

Fig. 1. Schematic of the beam transformer resonator. 
incidence and mirror radii are selected so that $R_{A} \sin \left(\theta_{A}\right) \tan \left(\theta_{A}\right)=R_{B} \sin \left(\theta_{B}\right) \tan \left(\theta_{B}\right)$, then the two mirrors $(A, B)$ when separated by, $d=f_{A}{ }^{x}+f_{B}{ }^{x}=f_{A}{ }^{y}+f_{B}{ }^{y}$, satisfy the afocal condition for the orthogonal directions simultaneously. The resulting magnification factors of the beam intensity distribution in the orthogonal axes is given by; $\mathrm{M}^{\mathrm{x}}=\mathrm{f}_{\mathrm{B}}^{\mathrm{x}} / \mathrm{f}_{\mathrm{A}}{ }^{\mathrm{x}}=\left(\mathrm{R}_{\mathrm{A}} / \mathrm{R}_{\mathrm{B}}\right) \cdot\left(\cos \left(\theta_{\mathrm{A}}\right) \cos \left(\theta_{\mathrm{B}}\right)\right)$ and $\mathrm{M}^{\mathrm{y}}=\mathrm{f}_{\mathrm{B}}{ }^{\mathrm{y}} / \mathrm{f}_{\mathrm{A}}{ }^{\mathrm{y}}=\left(\mathrm{R}_{\mathrm{A}} / \mathrm{R}_{\mathrm{B}}\right) /\left(\cos \left(\theta_{\mathrm{A}}\right) \cos \left(\theta_{\mathrm{B}}\right)\right)$. Noting that the stability criterion for an active resonator must be satisfied, intracavity the choice of mirror separation differs from that shown. Applying this concept allows an elliptical cavity mode in the slab to be transformed such that it is circular exiting the mirror pair, dependent upon the choice of radii, the tilt angles, and their respective spacing. An analogous optical system would be two orthogonal cylindrical telescopes with their magnifying directions opposing each other, which has the disadvantage of eight lens interface losses and relatively large phase aberration, as opposed to only two reflections for a beam transforming spherical mirror pair.

\section{Experiment results and discussion}

Fig. 1 is a schematic of the experimental setup used, comprising, a single $792 \mathrm{~nm}$ diode-bar end-pumping a 1x10x12mm, a-cut, 0.5 at. \% Nd:YLF slab (c-axis aligned to $\mathrm{x}$ axis) and the beam transformer resonator with a $10 \% \mathrm{~T}$ output coupler (OC) mirror. Heatsinks provided uniform heat extraction through the top and bottom slab surfaces. Pump absorption was $94 \%$ with an in-slab average beam size of $\sim 4250 \mu \mathrm{m}$ x $425 \mu \mathrm{m}$.

Laser threshold occurred at an incident pump power of $3.6 \mathrm{~W}$ consistent with theoretical calculations and the estimated round-trip cavity losses (L 2.8\%). Due to a lower p-pol. reflectance (w.r.t. s-pol.) for the tilted mirrors, a half waveplate was used to rotate the polarisation between mirrors A and B. At the maximum available incident pump power of $30 \mathrm{~W}$ an output power of $8 \mathrm{~W}$ was obtained, with a slope efficiency of $33 \%(35 \%)$ with respect to incident (absorbed) power. Relay-imaging the output coupler with a telescope, the second moments radii of the exiting beam were determined to be $\sim 0.4 \mathrm{~mm}$ for both axes with measured beam quality of $\mathrm{M}_{\mathrm{x}}{ }^{2}=1.2$ and $\mathrm{M}_{\mathrm{y}}{ }^{2}=1.4$.

Results of a model for the cavity-mode size as a function of the position from the HR mirror are shown in Fig. 2, illustrating the large change in aspect ratio obtained between the slab and output coupler. Future investigation of this resonator concept will allow further optimisation of the intra-cavity mode dimensions to enable diffractionlimited performance with the appropriate aspect ratio for a particular pump distribution.

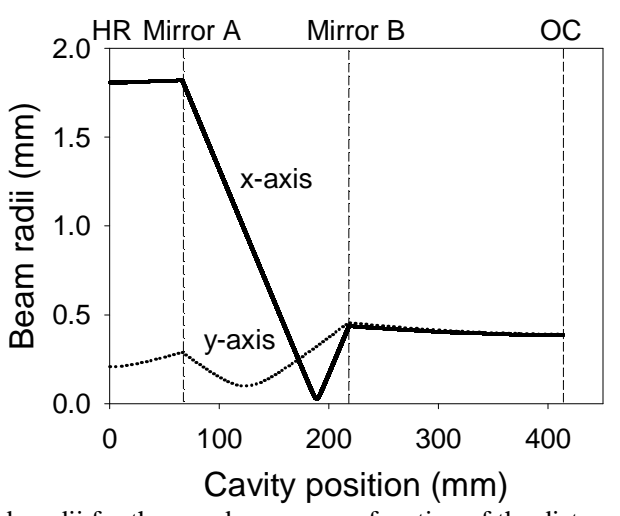

Fig. 2. Plot of the intra-cavity mode radii for the $x$ and $y$-axes as a function of the distance from the HR mirror.

\section{Summary}

We have demonstrated a novel laser resonator that provides an order of magnitude change in the aspect ratio of the intracavity mode, between the gain medium and the output coupler. Good conversion efficiency and beam quality demonstrate that this concept is ideally suited to planar gain media with highly elliptical gain distributions, fully exploiting the thermal advantages of this geometry. This approach opens the way for power-scalable solutions for pulsed as well as CW lasers, a unique advantage of thin-planar media over the fibre or thin-disc laser architectures.

\section{References}

[1] J. Harrison, P. F. Moulton, and G. A. Scott, "13-W, M2<1.2 Nd:YLF Laser Pumped by a Pair of 20-W Diode-Laser Bars," Conference on Lasers and Electro Optics - Postdeadline papers (CLEO '95), CPD20 (1995)

[2] R. Paschotta, J. Aus der Au, and U. Keller, "Thermal effects in high-power end-pumped lasers with elliptical-mode geometry," IEEE Journal of Selected Topics in Quantum Electronics 6, 636-642 (2000) 